\title{
Civil Rights Songs: A Chronological Listing
}

\section{LEONARD LEHRMAN}

This Civil Rights Song List is not a comprehensive catalogue. This list is merely the culmination of a set of lists that I will continue to augment on my own as more songs are created or discovered and added. The data presented here were amassed as a special project during the COVID-19 pandemic and as part of my duties as reference librarian at Oyster Bay-East Norwich Public Library in Oyster Bay, NY. Any opinions expressed in this article are my own and do not represent those of the library, its staff, or the Board of Trustees.

My own interest in civil rights, and in the power of music, dates back to the early 1960s, when my mother took me to a "Ban the Bomb" demonstration at the United Nations, and my father took me to the August 28, 1963, March on Washington. Their examples and these events would inspire a life of activism expressed through music, including my first musical on atomic testing and imperialism. I also formed the Metropolitan Philharmonic Chorus in 1988 to perform works no other chorus would or could do, including the cantata I Have A Dream written by my teacher and mentor Elie Siegmeister and performed in its Manhattan premiere with William Warfield on January 15, 1989, at the Harlem School of the Arts. The attendance of Pete Seeger at that performance prompted him to approach me, leading to wonderful collaborative opportunities in workshops, performances, arrangements, and compositions.

This project is intended as a starting point for others to build on. The list appended to this article is merely a snapshot in time of both a work in progress and, so to speak, a work about progress—social progress. Viewed chronologically, these songs have much to tell about the history of our country-and a few others, as well. Strictly speaking, the list is not exactly chronological: precise months and dates are not taken into account; only years, with titles alphabetized within each year. They begin half a millennium ago, and include every year since 1930 (except 1944, 1950, 1952, 1955, 1961, 1986-7, 1995, and 1997).

The names most frequently recurring here are: Bob Dylan (9); Pete Seeger and Bob Marley (8); Si Kahn and Langston Hughes (7); Elie Siegmeister and Phil Ochs (6); Bernice Johnson Reagon, Marvin Gaye, Tom Paxton, Peace Poets, Public Enemy, Tupac Shakur, and Nina Simone (5); Charon Hribar, Kendrick Lamar, Curtis Mayfield, Earl Robinson, and Staple Singers (4); Yara Allen, Marc Blitzstein, Oscar Brown, Jr., Mos Def, Anne Feeney, Rhiannon Giddens, Bertha Gober, Woody Guthrie, Jay-Z, Lead Belly, Tom Lehrer, Gil Scott-Heron, Barrett Strong, Kanye West, and Norman J. Whitfield (3). ${ }^{1}$

Many songs are associated more with their performers than with the writers and composers who created them, the best example being "Strange Fruit," which is often (mis)attributed to Billie Holiday, though it was written by Abel Meeropol under the pen-name Lewis Allan. Likewise, Lesley Gore is most closely associated with "You Don't Own Me," though it was written by John Madara and David White. Many of the songs Elvis Presley sang were also co-copyrighted by him, though he wrote none of them, and he is therefore not listed here. And, of course, "cover" artists like Joan Baez, Judy Collins, and Mara Levine

\footnotetext{
${ }^{1}$ My own name appears quite a few times as well since, as a composer, I know my own works catalog more thoroughly than anyone else's.
} 
often sing songs by writers like Phil Ochs, Bob Dylan, and Malvina Reynolds, sometimes bringing out the lyricism of the melodies even more than their creators did.

In defining "song" for this list, I included conventional settings of music for voice(s) with or without accompaniment, chants, talking blues, rap, hip-hop, and some hymns. Purely instrumental pieces, even with political titles, and even if called "songs without words," are not found here.

Then there is the question of the meaning of "civil rights." A dictionary definition includes the rights "to political and social freedom and equality." In general parlance, the term also denotes struggling against racism, prejudice and bigotry. But no matter how political, or relevant, or powerful, or good, not every song of struggle, labor, prison, protest, peace, or even identity-racial or otherwise-is necessarily a civil rights song. Most of the great Spanish Civil War songs are not conventionally considered civil rights songs (even if many have been called "Songs for Democracy"), but I did include Tom Lehrer's lampoon of them, because of his message, even though satirical, on "the fight against poverty, war and 'injestice." Likewise, I included songs about freedom, slavery, abolition, and the Underground Railroad. Spirituals and religious songs are also on this list if they have been adapted for use in the struggle for civil rights.

The list contains a number of songs that have been repurposed and refocused by adaptation and parody. Many of our nation's most important songs are indeed parodies or contain satire as a kind of critique, including "America” ("God Save the King”), "Battle Hymn of the Republic" ("John Brown's Body," and earlier than that, probably some revival hymns), and "This Land Is Your Land" (a Carter Family tune). Two songs of earlier generations, "Michael, Row the Boat Ashore" and "We're Marching to Pretoria," became adapted as civil rights songs in the 1950s, promoted especially by the Weavers. The first was discovered by Tony Saletan in the Boston Public Library, with roots in the Underground Railroad. The second was actually a British Army song from the Boer War, whose repurposing may be viewed retrospectively as somewhat humorous but effective nonetheless.

Quite a few Broadway shows and songs have dealt with civil rights, at least obliquely, such as Finian's Rainbow in 1947; "Carefully Taught" in Rodgers and Hammerstein's 1949 South Pacific, "Simple," which refers to the NAACP, in Anyone Can Whistle by Stephen Sondheim; and as an important theme in Hair, Ragtime, and Raisin-Judd Woldin's musicalization of Lorraine Hansberry's classic play A Raisin in the Sun. The earliest Broadway civil rights song, though, did not begin as one. Paul Robeson transformed "Ol' Man River" in Jerome Kern's and Oscar Hammerstein's Showboat of 1927 from a plaint of resignation into a fighting anthem during the Spanish Civil War, ten years later. Robeson also championed John LaTouche's and Earl Robinson's 1939 "Ballad for Americans" and Marc Blitzstein's 1941 song "Purest Kind of a Guy," and was particularly fond of its line "black or white or tan ..." (as attested to by Blitzstein's sister, Josephine Davis, in interviews with me and Blitzstein biographer Eric Gordon).

Other songs mention civil rights specifically, like Tom Paxton's “Daily News” or Joel Mandelbaum's and Leah Fichandler's "The Causes Are Waiting for You." Many recent songs have tended toward street chants-for instance, those at Black Lives Matter marches. ${ }^{2}$ But I have also tried to include songs from operas and oratorios, such as those about Dr. King, the Freedom Riders, Emmett Till, Medgar Evers, the three civil rights workers killed in Mississippi in 1964, and many more about recent victims like George Floyd, Breonna Taylor, and Michael Brown.

During the 2020 pandemic, the York Theatre sponsored a seven-round contest for songwriters on a

\footnotetext{
${ }^{2}$ On June 19, 2020, I joined and spoke at one such march, recalling words I had heard Dr. Martin Luther King, Jr., say in Washington and in Great Neck.
} 
different theme every two weeks. In Week 4, in response to Black Lives Matter, the theme was "What Matters to You." A few interesting civil rights songs resulted from that assignment. I composed seven songs for the first six rounds, perhaps the most memorable being a collaboration with Suffolk County's first poet laureate, George Wallace (not to be confused with the late governor of Alabama, about whom there have also been quite a few civil rights songs written). An excerpt from George's text reads:

we can all fit within

we can all do without

but don't push outsiders in

by tossing insiders out

fight for your rights as a minority

but don't dis your allies in the majority

In eschewing capital letters, George admitted to having been influenced by E. E. Cummings, and one of Cummings's poems also made it into this list—a 1926 anti-war send-up of political hypocrisy, in a couple of settings (mine of 1982; Declan McKenna's of 2016). The most frequently occurring of any of the writers is Langston Hughes. Hughes's poems have also been a source of inspiration for quite a few composers, most notably Elie Siegmeister, who set more of his poetry than anyone else. The Hughes poem that seems to have been set by more composers than any other in my lists (eight in total) is "Dreams," which arguably inspired Dr. King, about whom more civil rights songs seem to have been written than anyone else.

Civil rights songs are constantly being written and could be added to similar lists of your own making. At the time of this writing, I think this is the largest list of its kind (522 items) and so a significant foundation for researchers and others. I encourage anyone who wants to take up the mantle to continue the work. 


\section{Chronological List of Civil Rights Song Titles and Creators}

(with thanks to Zalmen Mlotek, NYTF; Bennet Zurofsky, Philip Aaberg, and Si Kahn)

\begin{tabular}{|c|c|c|}
\hline $1500 \mathrm{~s}$ & De Colores & $\begin{array}{l}\text { Spanish folk; adapted by Cursillos in Majorca, } \\
1944\end{array}$ \\
\hline $1700 \mathrm{~s}$ & $\begin{array}{l}\text { (There's a) Better Day A- } \\
\text { Coming }\end{array}$ & Folk; adapted by Michel LaRue, 1960 \\
\hline $\begin{array}{l}1779 \text { (published } \\
\text { as a hymn) }\end{array}$ & Amazing Grace & words: John Newton, 1772; tune: "New Britain" \\
\hline $1800 \mathrm{~s}$ & $\begin{array}{l}\text { Mir viln frayhayt [We Want } \\
\text { Freedom] }\end{array}$ & Anonymous; tune: "Sholom Aleichem" \\
\hline 1824 & Ode to Joy & $\begin{array}{l}\text { words: from the poem An die Freude by } \\
\text { Friedrich Schiller, 1785; music: Ludwig van } \\
\text { Beethoven for his Ninth Symphony, 1824; sung } \\
\text { by Paul Robeson for Welsh miners, 1957; sung by } \\
\text { Chilean protesters and as Anthem of Europe, } \\
\text { 1972; performed as An die Freiheit ("Ode to } \\
\text { Freedom") under Leonard Bernstein at the fall of } \\
\text { the Berlin Wall, 1989; adapted by the Deadly } \\
\text { Snakes, } 2003\end{array}$ \\
\hline 1825 & We Are Climbing Jacob's Ladder & Spiritual \\
\hline 1842 & $\begin{array}{l}\text { The Abolitionist Hymn [a.k.a. } \\
\text { Prayer of the Abolitionist] }\end{array}$ & John Pierpont (tune: "Old Hundredth") \\
\hline $1841-3$ & I Am an Abolitionist & William Lloyd Garrison (tune: “Auld Lang Syne’) \\
\hline 1842 & Die Gedanken sind frei & Hoffmann von Fallersleben \\
\hline 1844 & Get Off the Track! & Jesse Hutchinson, Jr. (tune: “Old Dan Tucker”) \\
\hline 1844 & Oh Freedom! & $\begin{array}{l}\text { Slave song; adapted by John Handcox, 1935; } \\
\text { marching song of Atlanta race riots, 1906; sung } \\
\text { and then recorded by Odetta, 1944, } 1956\end{array}$ \\
\hline 1847 & O Holy Night & $\begin{array}{l}\text { words: Placide Cappeau; music: Adolphe Adam } \\
\text { (English translation: John Sullivan Dwight, 1855) }\end{array}$ \\
\hline $\begin{array}{l}\text { 1850s (exact date } \\
\text { unknown) }\end{array}$ & No Irish Need Apply & Anonymous \\
\hline 1855 (published) & Slavery Is a Hard Foe to Battle & $\begin{array}{l}\text { words: Judson Hutchison; tune: "Jordan Is a } \\
\text { Hard Road to Travel” by Dan Emmett, } 1853\end{array}$ \\
\hline $\begin{array}{l}\text { 1866-92 (exact } \\
\text { date unknown) }\end{array}$ & $\begin{array}{l}\text { In Ale Gasn-Hey, Hey, Daloy } \\
\text { Politzey [Everywhere You } \\
\text { Look-Down with the Police!] }\end{array}$ & $\begin{array}{l}\text { David Edelstadt (tune includes quote from } \\
\text { Russian folk song parodied as "Tsar Nikolai izdal } \\
\text { manifest: Myortvym svoboda, zhivikh pod } \\
\text { arrest!" ["Tsar Nicholas issued a manifesto: } \\
\text { Freedom for the dead; the living under arrest!"] } \\
\text { quoted in Leonard Lehrman's opera Sima, 1976 }\end{array}$ \\
\hline $\begin{array}{l}\text { 1899-1966 (exact } \\
\text { date unknown) }\end{array}$ & $\begin{array}{l}\text { Maknes Geyen [Masses } \\
\text { Marching] }\end{array}$ & Mikhl Gelbart \\
\hline $1860 \mathrm{~s}$ & $\begin{array}{l}\text { No More Auction Block (for } \\
\text { Me) [a.k.a. Many Thousand } \\
\text { Gone] }\end{array}$ & Civil War song \\
\hline 1860 & Song of the Free & $\begin{array}{l}\text { Underground Railroad Song (tune: "Oh! } \\
\text { Susanna") }\end{array}$ \\
\hline
\end{tabular}




\begin{tabular}{|c|c|c|}
\hline 1861 & $\begin{array}{l}\text { John Brown's Body [a.k.a. The } \\
\text { John Brown Song] }\end{array}$ & $\begin{array}{l}\text { Anonymous (tune: "Say, Brothers Will You Meet } \\
\text { Us?") }\end{array}$ \\
\hline pre-1862 & Steal Away & Spiritual \\
\hline 1862 & $\begin{array}{l}\text { The Battle Cry of Freedom } \\
\text { [a.k.a. Rally 'Round the Flag] }\end{array}$ & George Frederick Root \\
\hline 1862 & $\begin{array}{l}\text { Battle Hymn of the Republic } \\
\text { [a.k.a. Mine Eyes Have Seen the } \\
\text { Glory] }\end{array}$ & Julia Ward Howe (tune: “John Brown’s Body”) \\
\hline 1862 (published) & Go Down, Moses & $\begin{array}{l}\text { Spiritual (arrangements: Henry Burleigh, } \\
\text { Florence Price) }\end{array}$ \\
\hline 1863 & $\begin{array}{l}\text { Get on Board [a.k.a. Get on } \\
\text { Board, Little Children; a.k.a. The } \\
\text { Gospel Train] }\end{array}$ & sometimes credited to John Chamberlain \\
\hline 1864 & Wake Nicodemus & Henry Clay Work \\
\hline 1865 & $\begin{array}{l}\text { Slavery Chain Done Broke at } \\
\text { Last }\end{array}$ & Spiritual (tune: "Joshua Fit de Battle of Jericho") \\
\hline 1867 (published) & Michael, Row the Boat Ashore & $\begin{array}{l}\text { Spiritual (published in Slave Songs of the United } \\
\text { States) }\end{array}$ \\
\hline 1880 & We Are Soldiers in the Army & Anonymous \\
\hline $\begin{array}{l}\text { 1856-1932 (exact } \\
\text { date unknown) }\end{array}$ & $\begin{array}{l}\text { Ale Brider [a.k.a. Un mir zaynen } \\
\text { ale brider (And We Are All } \\
\text { Brothers)] }\end{array}$ & Morris Winchevsky \\
\hline $\begin{array}{l}\text { 1856-1932 (exact } \\
\text { date unknown) }\end{array}$ & $\begin{array}{l}\text { O di velt vet vern yinger [a.k.a. } \\
\text { Di Zukunft (The Future)] }\end{array}$ & Morris Winchevsky \\
\hline 1900 & Lift Every Voice and Sing & James Weldon Johnson \\
\hline 1907 & Will the Circle Be Unbroken? & $\begin{array}{l}\text { words: Ada R. Habershon; music: Charles H. } \\
\text { Gabriel }\end{array}$ \\
\hline 1915 & Solidarity Forever & Ralph Chaplin (tune: “John Brown’s Body”) \\
\hline 1917 & Bread and Roses & $\begin{array}{l}\text { words: James Oppenheim, 1911; music: Caroline } \\
\text { Kohlsaat, } 1917\end{array}$ \\
\hline 1917 & I Don't Feel No-Ways Tired & Spiritual (arrangement: Harry Thacker Burleigh) \\
\hline 1917 & Walk Together, Children & Spiritual (arrangement: J. Rosamond Johnson) \\
\hline 1918 (published) & Down by the Riverside & Spiritual \\
\hline $1920 \mathrm{~s}$ & This Little Light of Mine & adapted by Harry Dixon Loes \\
\hline 1922 & This Train (Is Bound for Glory) & Gospel \\
\hline 1924 & $\begin{array}{l}\text { The Prisoner's Song (If I Had } \\
\text { the Wings of an Angel) }\end{array}$ & $\begin{array}{l}\text { Robert and Guy Massey; copyrighted by Vernon } \\
\text { Dalhart }\end{array}$ \\
\hline 1927 & Georgia Stockade Blues & Sara Martin \\
\hline 1927 (published) & $\begin{array}{l}\text { He's Got the Whole World in } \\
\text { His Hands }\end{array}$ & Spiritual \\
\hline 1928 (published) & Follow the Drinkin' Gourd & Spiritual (Underground Railroad song) \\
\hline 1930 (composed) & $\begin{array}{l}\text { How Can You Keep on Movin' } \\
\text { (Unless You Migrate Too) }\end{array}$ & Agnes "Sis" Cunningham (published in 1959) \\
\hline 1931 & $\begin{array}{l}\text { I'll Be Glad When You're Dead } \\
\text { (You Rascal You) }\end{array}$ & Sam Theard; adapted by Louis Armstrong \\
\hline 1931 & I'll Fly Away & $\begin{array}{l}\text { Albert Edward Brumley, influenced by “The } \\
\text { Prisoner's Song” }\end{array}$ \\
\hline 1932 & Brother, Can You Spare a Dime? & $\begin{array}{l}\text { from Americana; words: E.Y. "Yip" Harburg; } \\
\text { music: Jay Gorney }\end{array}$ \\
\hline 1932 & Which Side Are You On? & Florence Reece; later adapted by Ani DiFranco, et al \\
\hline
\end{tabular}




\begin{tabular}{|c|c|c|}
\hline 1933 & Supper Time & Irving Berlin (written for Ethel Waters) \\
\hline 1934 & Park Bench & words: Langston Hughes; music: Elie Siegmeister \\
\hline 1934 & $\begin{array}{l}\text { The Scottsboro Boys Shall Not } \\
\text { Die }\end{array}$ & $\begin{array}{l}\text { words: Charles Abron; music: Elie Siegmeister } \\
\text { (a.k.a. L. E. Swift) }\end{array}$ \\
\hline 1935 & We Shall Not Be Moved & Textile Workers; many adaptations, thru present \\
\hline 1936 & Joe Worker & from The Cradle Will Rock, Marc Blitzstein \\
\hline 1937 & The Bourgeois Blues & Huddie Ledbetter (a.k.a. Lead Belly) \\
\hline 1937 & Ol' Man River & $\begin{array}{l}\text { from Show Boat, 1927; words: Oscar } \\
\text { Hammerstein; music: Jerome Kern; version } \\
\text { adapted \& sung by Paul Robeson, } 1937\end{array}$ \\
\hline 1937 & Strange Fruit & Abel Meeropol (a.k.a. Lewis Allan) \\
\hline 1938 & Certainly Lord & $\begin{array}{l}\text { Spiritual (used in Regina by Marc Blitzstein, } \\
\text { 1949) }\end{array}$ \\
\hline 1938 & Joe Hill & words: Alfred Hayes; music: Earl Robinson \\
\hline 1938 & $\begin{array}{l}\text { Precious Lord, Take My Hand } \\
\text { [a.k.a. Take My Hand, Precious } \\
\text { Lord] }\end{array}$ & Thomas A. Dorsey; Dr. King’s favorite hymn \\
\hline 1939 & $\begin{array}{l}\text { Ballad for Americans [a.k.a. The } \\
\text { Ballad for Uncle Sam] }\end{array}$ & $\begin{array}{l}\text { from Sing for Your Supper, words: John } \\
\text { LaTouche; music: Earl Robinson }\end{array}$ \\
\hline 1939 & Free at Last & $\begin{array}{l}\text { Spiritual (recorded by the Blind Boys of Alabama, } \\
\text { 1939); quoted in "I Have a Dream" speech by } \\
\text { M. L. King, Jr.; quoted in I Have A Dream } \\
\text { Cantata by Elie Siegmeister, 1966-7; quoted in I } \\
\text { Have a Dream Oratorio by James Furman, 1970-1 }\end{array}$ \\
\hline $\begin{array}{l}\text { 1939-47 } \\
\text { (several versions) }\end{array}$ & $\begin{array}{l}\text { There's a Man Goin' Round } \\
\text { Takin' Names }\end{array}$ & Huddie Ledbetter (a.k.a. Lead Belly) \\
\hline 1940 & Another Man Done Gone & (collected by John and Alan Lomax) \\
\hline 1940 & $\begin{array}{l}\text { Jim Crow (Lincoln Set the } \\
\text { Negro Free; Why Is He Still in } \\
\text { Slavery?) }\end{array}$ & $\begin{array}{l}\text { The Almanac Singers (Woody Guthrie, Pete } \\
\text { Seeger, Millard Lampell) }\end{array}$ \\
\hline 1940 (published) & $\begin{array}{l}\text { Soon I Will Be Done (Gonna } \\
\text { Turn the World Around) }\end{array}$ & Spiritual (adapted 2001) \\
\hline 1940 & This Land Is Your Land & $\begin{array}{l}\text { Woody Guthrie (tune: "When the World's on } \\
\text { Fire" by the Carter Family) }\end{array}$ \\
\hline 1941 & The Purest Kind of a Guy & from No for an Answer, Marc Blitzstein \\
\hline 1941 & Uncle Sam Says & Josh White \\
\hline 1942 & All You Fascists Bound to Lose & Woody Guthrie \\
\hline 1942 & $\begin{array}{l}\text { I'm On My Way (to [the] } \\
\text { Freedom Land) }\end{array}$ & $\begin{array}{l}\text { Mamie Brown and Carlton Reece (arrangement: } \\
\text { Arthur Stern) }\end{array}$ \\
\hline 1943 & The House I Live In & $\begin{array}{l}\text { words: Abel Meeropol (a.k.a. Lewis Allan); music: } \\
\text { Earl Robinson }\end{array}$ \\
\hline 1945 & Hold Fast to Dreams & $\begin{array}{l}\text { words: Langston Hughes, 1922; music: Florence } \\
\text { Price }\end{array}$ \\
\hline 1946 & Hallelujah, I'm A-Travelin' & Anonymous (tune: "Hallelujah, I'm a Bum”) \\
\hline 1947 & Duncan and Brady & Huddie Ledbetter (a.k.a. Lead Belly) \\
\hline 1947 & The Freedom Train & Irving Berlin \\
\hline 1947 & (I Will) Move on Up a Little Higher & W. Herbert Brewster \\
\hline 1947 & Talking Union & Millard Lampell, Lee Hays, and Pete Seeger \\
\hline 1947 & $\begin{array}{l}\text { When the Idle Poor Become the } \\
\text { Idle Rich }\end{array}$ & $\begin{array}{l}\text { from Finian’s Rainbow, words: E. Y. "Yip" } \\
\text { Harburg; music: Burton Lane }\end{array}$ \\
\hline
\end{tabular}




\begin{tabular}{|c|c|c|}
\hline 1948 & Black, Brown and White [Blues] & William “Big Bill” Broonzy \\
\hline 1948 & Brown-Skinned Cow & Hy Zaret and Lou Singer \\
\hline 1948 & Passing Through & Dick Blakeslee \\
\hline 1949 & $\begin{array}{l}\text { If I Had a Hammer [a.k.a. The } \\
\text { Hammer Song] }\end{array}$ & Lee Hays and Pete Seeger \\
\hline 1949 & $\begin{array}{l}\text { You've Got to Be Carefully } \\
\text { Taught }\end{array}$ & $\begin{array}{l}\text { from South Pacific, words: Oscar Hammerstein; } \\
\text { music: Richard Rodgers }\end{array}$ \\
\hline 1951 & Chalk Marks on the Sidewalk & words: Langston Hughes; music: Elie Siegmeister \\
\hline 1951 & How I Got Over & Clara Ward \\
\hline 1953 & I Wanna Go Back to Dixie & Tom Lehrer \\
\hline 1953 & We Are Marching to Pretoria & $\begin{array}{l}\text { Boer War Song, 1900; adapted by The Weavers, } \\
1953\end{array}$ \\
\hline 1954 & Michael, Row the Boat Ashore & $\begin{array}{l}\text { Slave song (first published in 1867); adapted by } \\
\text { Tony Saletan with harmony and some lyrics } \\
\text { changes }\end{array}$ \\
\hline 1956 & Black and White & Earl Robinson \\
\hline 1956 & Brown Eyed Handsome Man & Chuck Berry \\
\hline 1956 & Union Maid & Woody Guthrie (tune: “Red Wing”) \\
\hline 1957 & Wasn't That a Time? & Lee Hays \\
\hline 1958 & Turn, Turn, Turn & Pete Seeger (words from Ecclesiastes 3:1-8) \\
\hline 1959 & Fables of Faubus & Charles Mingus \\
\hline 1959 & No Crime, No Law & Lord Commander \\
\hline $\begin{array}{l}\text { 1960s (exact date } \\
\text { unknown) }\end{array}$ & $\begin{array}{l}\text { Calypso Freedom [a.k.a. } \\
\text { Freedom's Coming and It Won't } \\
\text { Be Long] }\end{array}$ & $\begin{array}{l}\text { Willie Peacock (tune: "Banana Boat Song [a.k.a. } \\
\text { Day-O]") }\end{array}$ \\
\hline $\begin{array}{l}\text { 1960s (exact date } \\
\text { unknown) }\end{array}$ & $\begin{array}{l}\text { Everybody Says [Wants] } \\
\text { Freedom }\end{array}$ & $\begin{array}{l}\text { Spiritual (recorded on Freedom Songs: Selma } \\
\text { Alabama, 1965, Smithsonian Folkways) }\end{array}$ \\
\hline $\begin{array}{l}\text { 1960s (exact date } \\
\text { unknown) }\end{array}$ & Going Down to Mississippi & Phil Ochs \\
\hline $\begin{array}{l}\text { 1960s (exact date } \\
\text { unknown) }\end{array}$ & Governor Wallace & Freedom Singers \\
\hline $\begin{array}{l}\text { 1960s (exact date } \\
\text { unknown) }\end{array}$ & $\begin{array}{l}\text { Guide My Feet (While I Run } \\
\text { This Race) }\end{array}$ & $\begin{array}{l}\text { Spiritual (tune: "Do Lord Remember Me," } \\
\text { attributed to Julia Ward Howe, 1860s) }\end{array}$ \\
\hline $\begin{array}{l}\text { 1960s (exact date } \\
\text { unknown) }\end{array}$ & $\begin{array}{l}\text { Lord, Hold My Hand While I } \\
\text { Run This Race }\end{array}$ & Spiritual \\
\hline $\begin{array}{l}\text { 1960s (exact date } \\
\text { unknown) }\end{array}$ & Oh Pritchett, Oh Kelly & $\begin{array}{l}\text { Bertha Gober, Rutha Harris, Charles Sherrod, } \\
\text { and Jamie Culbreath (tune: "Rockin' Jerusalem") }\end{array}$ \\
\hline $\begin{array}{l}\text { 1960s (exact date } \\
\text { unknown) }\end{array}$ & $\begin{array}{l}\text { Over My Head I See Freedom in } \\
\text { the Air }\end{array}$ & Bernice Johnson Reagon \\
\hline $\begin{array}{l}\text { 1960s (exact date } \\
\text { unknown) }\end{array}$ & Walk with Me, Lord & Spiritual; adapted by Fannie Lou Hamer \\
\hline 1960 & $\begin{array}{l}\text { (There's a) Better Day A- } \\
\text { Coming }\end{array}$ & adapted by Michel LaRue from a 1700s tune \\
\hline 1960 & Brown Baby & Oscar Brown, Jr. \\
\hline 1960 & $\begin{array}{l}\text { Dog, Dog [a.k.a. Dogs; a.k.a. } \\
\text { Your Dog Loves My Dog] }\end{array}$ & Freedom Singers \\
\hline 1960 & Freedom Day & words: Oscar Brown; music: Max Roach \\
\hline 1960 & $\begin{array}{l}\text { Greensboro [a.k.a. Ballad of the } \\
\text { Student Sit-Ins] }\end{array}$ & Guy Caravan, Eve Merriam, and Norma Curtis \\
\hline 1960 & Chain Gang & Sam Cooke \\
\hline
\end{tabular}




\begin{tabular}{|c|c|c|}
\hline 1960 & We Are Moving On to Vict'ry & Pete Seeger \\
\hline $\begin{array}{l}\text { 1946-60 } \\
\text { (several versions) }\end{array}$ & We Shall Overcome & $\begin{array}{l}\text { Zilphia Horton, Frank Hamilton, Guy Caravan, } \\
\text { and Pete Seeger (original hymn: "I'll Overcome } \\
\text { Some Day" by Charles Albert Tindley, published } \\
\text { 1901; published as "We Will Overcome" in } \\
\text { People's Song Bulletin, 1945) }\end{array}$ \\
\hline 1962 & $\begin{array}{l}\text { Ain't Gonna Let Nobody Turn } \\
\text { Me Round [a.k.a. Don't Let } \\
\text { Nobody Turn You Around] }\end{array}$ & Spiritual \\
\hline 1962 & Blowin' in the Wind & Bob Dylan \\
\hline 1962 & $\begin{array}{l}\text { The Death [a.k.a. Ballad] of } \\
\text { Emmett Till }\end{array}$ & Bob Dylan \\
\hline 1962 & Freedom in the Air & Bernice Johnson Reagon \\
\hline 1962 & Hymn to Freedom & Oscar Peterson \\
\hline 1962 & Ride On Red, Ride On & Louisiana Red \\
\hline 1963 & Ain't A-Scared of Your Jail & Lester Cobb (tune: “Old Grey Mare”) \\
\hline 1963 & Been Down into the South & Bob Zellner \\
\hline 1963 & $\begin{array}{l}\text { If You Miss Me at the Back of } \\
\text { the Bus }\end{array}$ & $\begin{array}{l}\text { Charles Neblett (tune: "O Mary, Don't You } \\
\text { Weep") }\end{array}$ \\
\hline 1963 & Get Your Rights, Jack & CORE Freedom Singers \\
\hline 1963 & $\begin{array}{l}\text { Go Tell It on the Mountain (to } \\
\text { Let My People Go!) }\end{array}$ & $\begin{array}{l}\text { Spiritual (published 1909); adapted Fannie Lou } \\
\text { Hamer }\end{array}$ \\
\hline 1963 & $\begin{array}{l}\text { I Wish I Knew How It Would } \\
\text { Feel to Be Free }\end{array}$ & Billy Taylor and Dick Dallas \\
\hline 1963 & In the Upper Room & L. E. Campbell \\
\hline 1963 & Oginga Odinga & Matthew Jones \\
\hline 1963 & Only a Pawn in Their Game & Bob Dylan \\
\hline 1963 & Power and the Glory & Phil Ochs \\
\hline 1963 & Stone Walls and Steel Bars & Ray Pennington and Roy Marcum \\
\hline 1963 & There but for Fortune & Phil Ochs \\
\hline 1963 & Uncle Tom's Prayer & Matthew Jones \\
\hline 1963 & We'll Never Turn Back & Bertha Gober \\
\hline 1963 & $\begin{array}{l}\text { Woke Up This Mornin' (with } \\
\text { My Mind Stayed on Freedom) }\end{array}$ & Robert Wesby \\
\hline 1963 & You Don't Own Me & John Madara and David White \\
\hline 1964 & Birmingham Sunday & Richard Fariña \\
\hline 1964 & Carry It On & Gil Turner and Marion Wade \\
\hline 1964 & A Change Is Gonna Come & Sam Cooke \\
\hline 1964 & Chimes of Freedom & Bob Dylan \\
\hline 1964 & Daily News & Tom Paxton \\
\hline 1964 & Dancin' in the Street(s) & $\begin{array}{l}\text { Marvin Gaye, William "Mickey" Stevenson, and } \\
\text { Ivy Jo Hunter }\end{array}$ \\
\hline 1964 & He Was My Brother & Paul Simon \\
\hline 1964 & $\begin{array}{l}\text { I'm Gonna Sit at the Welcome } \\
\text { Table }\end{array}$ & $\begin{array}{l}\text { Gospel hymn, adapted by McComb, MS, } \\
\text { Freedom School Students }\end{array}$ \\
\hline 1964 & In The Mississippi River & Marshall Jones \\
\hline 1964 & It Isn't Nice & Malvina Reynolds \\
\hline 1964 & Keep On Pushing & The Impressions \\
\hline 1964 & $\begin{array}{l}\text { The Lonesome Death of Hattie } \\
\text { Carroll }\end{array}$ & Bob Dylan \\
\hline
\end{tabular}




\begin{tabular}{|c|c|c|}
\hline 1964 & Mississippi Goddam & Nina Simone \\
\hline 1964 & Promised Land & Chuck Berry (tune: "Wabash Cannonball") \\
\hline 1964 & Simple & from Anyone Can Whistle; Stephen Sondheim \\
\hline 1964 & $\begin{array}{l}\text { The Times They Are A- } \\
\text { Changin' }\end{array}$ & Bob Dylan \\
\hline 1964 & $\begin{array}{l}\text { Too Many Martyrs [a.k.a. The } \\
\text { Ballad of Medgar Evers] }\end{array}$ & Phil Ochs \\
\hline 1964 & $\begin{array}{l}\text { What Did You Learn in School } \\
\text { Today? }\end{array}$ & Tom Paxton \\
\hline 1964 & When the Ship Comes In & Bob Dylan \\
\hline 1965 & Ain't That News? & Tom Paxton \\
\hline 1965 & Alabama Blues & J. B. Lenoir \\
\hline 1965 & Ballad of a Thin Man & Bob Dylan \\
\hline 1965 & Been in the Storm [So Long] & Folk \\
\hline 1965 & Days of Decision & Phil Ochs \\
\hline 1965 & The Folk Song Army & Tom Lehrer \\
\hline 1965 & Forty Acres and a Mule & Oscar Brown, Jr. \\
\hline 1965 & Four Women & Nina Simone \\
\hline 1965 & Freedom Is a Constant Struggle & Roberta Slavitt \\
\hline 1965 & Freedom Now Chant & $\begin{array}{l}\text { (recorded on Freedom Songs: Selma, Alabama, } \\
\text { 1965, Folkways Records) }\end{array}$ \\
\hline 1965 & $\begin{array}{l}\text { Goodman, Schwerner, and } \\
\text { Chaney }\end{array}$ & Tom Paxton \\
\hline 1965 & Here's to the State of Mississippi & Phil Ochs \\
\hline 1965 & $\begin{array}{l}\text { Keep Your Eyes on the Prize } \\
\text { [a.k.a. Hold On] }\end{array}$ & $\begin{array}{l}\text { Alice Wine (tune: "Keep Your Hand on the } \\
\text { Plow") }\end{array}$ \\
\hline 1965 & Maggie’s Farm & Bob Dylan \\
\hline 1965 & Michael, Andrew and James & Richard Fariña \\
\hline 1965 & National Brotherhood Week & Tom Lehrer \\
\hline $\begin{array}{l}\text { 1965-8 } \\
\text { (several versions) }\end{array}$ & Oh Wallace & James Orange \\
\hline 1965 & People Get Ready & Curtis Mayfield \\
\hline 1965 & Respect & Otis Redding \\
\hline 1965 & $\begin{array}{l}\text { Society's Child [a.k.a. Baby, I've } \\
\text { Been Thinking] }\end{array}$ & Janis Ian \\
\hline 1965 & Tell It On and On & $\begin{array}{l}\text { Edith Segal (on Goodman, Schwerner and } \\
\text { Chaney) }\end{array}$ \\
\hline 1965 (recorded) & Wade in the Water & $\begin{array}{l}\text { Spiritual (first published by Frederick J. Work and } \\
\text { John Wesley Work II in New Jubilee Songs as } \\
\text { Sung by the Fisk Jubilee Singers; recorded as a } \\
\text { civil rights song by the Staple Singers on the } \\
\text { album Freedom Highway) }\end{array}$ \\
\hline 1965 & We Didn't Know & $\begin{array}{l}\text { Tom Paxton; adapted by Leonard Lehrman, 2006, } \\
2019\end{array}$ \\
\hline 1965 & Why (Am I Treated So Bad)? & Staples Singers \\
\hline 1966 & $\begin{array}{l}\text { Ain't No Mountain High } \\
\text { Enough }\end{array}$ & Nickolas Ashford and Valerie Simpson \\
\hline 1966 & Burn, Baby, Burn & $\begin{array}{l}\text { Jimmy Collier and Frederick Douglass } \\
\text { Kirkpatrick }\end{array}$ \\
\hline 1966 & For What It's Worth & Stephen Stills \\
\hline
\end{tabular}




\begin{tabular}{|c|c|c|}
\hline 1966 & Those Three Are On My Mind & Pete Seeger and Frances Taylor \\
\hline 1966 & Compared to What? & Gene McDaniels \\
\hline 1967 & Black Boys-White Boys & $\begin{array}{l}\text { from Hair, words: Gerome Ragni and James } \\
\text { Rado; music: Galt MacDermot }\end{array}$ \\
\hline 1967 & $\begin{array}{l}\text { Exile; I Have a Dream; The } \\
\text { Sound of Freedom }\end{array}$ & $\begin{array}{l}\text { excerpts from Cantata, I Have a Dream; words by } \\
\text { Edward Mabley after speech by M.L. King; music } \\
\text { by Elie Siegmeister }\end{array}$ \\
\hline 1967 & The Motor City Is Burning & John Lee Hooker \\
\hline 1967 & Police on My Back & The Equals \\
\hline 1967 & Sittin' On the Dock of the Bay & Otis Redding \\
\hline 1967 & We're a Winner & Curtis Mayfield \\
\hline 1967 & What's Goin' On Down There? & Malvina Reynolds \\
\hline 1968 & Abraham, Martin and John & Dick Holler \\
\hline 1968 & Ballad of Martin Luther King & Mike Millius \\
\hline 1968 & Blackbird & John Lennon and Paul McCartney \\
\hline 1968 & Blues for Martin Luther King & Otis Spann \\
\hline 1968 & Everybody's Got a Right to Live & Frederick D. Kirkpatrick \\
\hline 1968 & Everyday People & Sly Stone \\
\hline 1968 & The Face of War & $\begin{array}{l}\text { title song in cycle of 5; words: Langston Hughes, } \\
\text { 1967; music: Elie Siegmeister, } 1968\end{array}$ \\
\hline 1968 & $\begin{array}{l}\text { I['ve] Been 'Buked and I['ve] } \\
\text { Been Scorned [We'll Never } \\
\text { Turn Back] }\end{array}$ & adapted by Bertha Gober \\
\hline 1968 & Piggies & George Harrison \\
\hline 1968 & $\begin{array}{l}\text { Say It Loud I'm Black and I'm } \\
\text { Proud }\end{array}$ & James Brown \\
\hline 1968 & Volunteered Slavery & Roland Kirk \\
\hline 1968 & Why? (The King of Love Is Dead) & Nina Simone \\
\hline 1969 & Freedom's Comin' & Charity \\
\hline 1969 & In the Ghetto & Mac Davis \\
\hline 1969 & $\begin{array}{l}\text { Have You Been to Jail for } \\
\text { Justice? }\end{array}$ & Anne Feeney \\
\hline 1969 & Is It Because I'm Black? & Syl Johnson \\
\hline 1969 & Message from a Black Man & Barrett Strong and Norman J. Whitfield \\
\hline 1969 & Rebuild America/Keep Hope Alive & Anne Feeney \\
\hline 1969 & Stand! & Sly Stone \\
\hline 1969 & To Be Young, Gifted and Black & words: Weldon Irvine; music: Nina Simone \\
\hline 1969 & $\begin{array}{l}\text { When Will We Be Paid? [for the } \\
\text { Work We've Done] }\end{array}$ & Staple Singers \\
\hline 1970 & $\begin{array}{l}\text { Ball of Confusion (That's What } \\
\text { the World Is Today) }\end{array}$ & Norman Whitfield and Barrett Strong \\
\hline 1970 & $\begin{array}{l}\text { (Don't Worry) If There's A Hell } \\
\text { Below We're All Going to Go }\end{array}$ & Curtis Mayfield \\
\hline 1970 & Down in Mississippi & J.B. Lenoir \\
\hline 1970 & $\begin{array}{l}\text { (For God's Sake) Give More } \\
\text { Power to the People }\end{array}$ & Eugene Record \\
\hline 1970 & Freedom Rider & Traffic \\
\hline 1970 & $\begin{array}{l}\text { I Don't Want Nobody to Give } \\
\text { Me Nothing (Open Up the } \\
\text { Door, I'll Get It Myself) }\end{array}$ & James Brown \\
\hline
\end{tabular}




\begin{tabular}{|c|c|c|}
\hline 1970 & No More & The Lumpen \\
\hline 1970 & Ohio & Crosby, Stills, Nash \& Young \\
\hline 1970 & Southern Man & Neil Young \\
\hline 1970 & $\begin{array}{l}\text { We Are the People [Who Are] } \\
\text { Darker Than Blue }\end{array}$ & Curtis Mayfield \\
\hline 1970 & When the Revolution Comes & The Last Poets \\
\hline 1970 & Yes, We Can & $\begin{array}{l}\text { Lee Dorsey and Allen Toussaint (adapted as "Yes, } \\
\text { We Can Can" by Allen Toussaint, 1973) }\end{array}$ \\
\hline $1970-1$ & Liberation/Poem & Sonia Sanchez \\
\hline 1971 & Freedom Song & Roberta Flack \\
\hline 1971 & Harlem River Drive & words: Calvin Clash; music: Eddie Palmieri \\
\hline 1971 & Law and Order & $\begin{array}{l}\text { from Inner City, words: Eve Merriam; music: } \\
\text { Helen Miller }\end{array}$ \\
\hline 1971 & Respect Yourself & The Staple Singers \\
\hline 1971 & $\begin{array}{l}\text { The Revolution Will Not Be } \\
\text { Televised }\end{array}$ & Gil Scott-Heron \\
\hline 1971 & Save the Children & Al Cleveland, Renaldo Benson, and Marvin Gaye \\
\hline 1971 & Smiling Faces Sometimes & Norman Whitfield and Barrett Strong \\
\hline 1971 & What's Going On? & Marvin Gaye \\
\hline 1971 & Wholy Holy & Al Cleveland, Renaldo Benson and Marvin Gaye \\
\hline 1972 & The Harder They Come & Jimmy Cliff \\
\hline 1972 & I'll Take You There & The Staple Singers \\
\hline 1972 & Inner City Blues & Marvin Gaye \\
\hline 1972 & No Knock & Gil Scott-Heron \\
\hline 1973 & Get Up, Stand Up & Bob Marley \\
\hline 1973 & Living for the City & Stevie Wonder \\
\hline 1973 & Me and Baby Brother & War \\
\hline 1973 & Measure the Valleys & $\begin{array}{l}\text { from Raisin; words: Robert Brittan (after Lorraine } \\
\text { Hansberry); music: Judd Woldin }\end{array}$ \\
\hline 1973 & Someday We'll All Be Free & Donny Hathaway \\
\hline 1974 & Bread and Roses & $\begin{array}{l}\text { words: James Oppenheim, 1911; music: Mimi } \\
\text { Fariña, } 1974\end{array}$ \\
\hline 1974 & Burn, Baby, Burn & Hudson Ford \\
\hline 1974 & Hercules & Aaron Neville \\
\hline 1974 & Revolution & Bob Marley \\
\hline 1974 & Sweet Home Alabama & Lynyrd Skynyrd \\
\hline 1974 & $\begin{array}{l}\text { Them Belly Full (But We } \\
\text { Hungry) }\end{array}$ & Bob Marley \\
\hline 1975 & Burnin' and Lootin' & Bob Marley \\
\hline 1975 & Chocolate City & Parliament \\
\hline 1975 & $\begin{array}{l}\text { Give the People What They } \\
\text { Want }\end{array}$ & The O'Jays \\
\hline 1975 & $\begin{array}{l}\text { The People United Will Never } \\
\text { Be Defeated! (El pueblo unido } \\
\text { jamás será vencido) }\end{array}$ & $\begin{array}{l}\text { variations by Frederic Rzewski of } 1973 \text { song by } \\
\text { Sergio Ortega and Quilapayún }\end{array}$ \\
\hline 1975 & Politicians in My Eyes & $\begin{array}{l}\text { Bobby, David, and Dannis Hackney (a.k.a. } \\
\text { "Death") }\end{array}$ \\
\hline 1976 & Joan [Joanne] Little & Bernice Johnson Reagon \\
\hline 1977 & Baltimore & Randy Newman \\
\hline 1977 & Equal Rights & Peter Tosh \\
\hline
\end{tabular}




\begin{tabular}{|c|c|c|}
\hline 1977 & Harriet Tubman & Walter Robinson \\
\hline 1977 & $\begin{array}{l}95 \text { South, All the Places We've } \\
\text { Been }\end{array}$ & Gil Scott-Heron and Brian Jackson \\
\hline 1977 & Sorrow, Tears and Blood & Fela Kuti \\
\hline 1978 & $\begin{array}{l}\text { Brot und Rosen [Bread and } \\
\text { Roses] }\end{array}$ & $\begin{array}{l}\text { words: James Oppenheim, 1911; translated into } \\
\text { German by Peter Maiwald; music: Renate Fresow }\end{array}$ \\
\hline 1978 & The Devil Went Down to Georgia & Charlie Daniels \\
\hline 1979 & Zimbabwe & Bob Marley \\
\hline $\begin{array}{l}\text { 1980s (exact date } \\
\text { unknown) }\end{array}$ & Courage [My Friend] & South African Anti-Apartheid song \\
\hline $\begin{array}{l}\text { 1980s (exact date } \\
\text { unknown) }\end{array}$ & Walking Down the Road & Si Kahn (written about South Africa) \\
\hline 1980 & Real Situation & Bob Marley \\
\hline 1980 & Redemption Song & Bob Marley \\
\hline 1980 & Yes, We Want Our Freedom & Cleo Kennedy and Carlton Reese \\
\hline 1981 & $\begin{array}{l}\text { Ella's Song-We Who Believe in } \\
\text { Freedom Cannot Rest Until It } \\
\text { Comes }\end{array}$ & $\begin{array}{l}\text { from the film Fundi; Bernice Johnson Reagon } \\
\text { (about Ella Baker) }\end{array}$ \\
\hline 1981 & Mr. Policeman & Rick James \\
\hline 1981 & Nazi Punks Fuck Off & Dead Kennedys \\
\hline 1981 & Rise Above & Black Flag \\
\hline 1982 & I Was Just a Stupid Dog to Them & Nina Simone \\
\hline 1982 & Know Your Rights & The Clash \\
\hline 1982 & "next to of course god" & $\begin{array}{l}\text { words: E. E. Cummings, 1926; music: Leonard } \\
\text { Lehrman }\end{array}$ \\
\hline 1983 & Batterram & Toddy Tee \\
\hline 1983 & The Causes Are Waiting for You & $\begin{array}{l}\text { words: Leah Fichandler; music; Joel } \\
\text { Mandelbaum; adapted by Leonard Lehrman, } 2007\end{array}$ \\
\hline 1983 & Chant Down Babylon & Bob Marley \\
\hline 1983 & I Am What I Am & from La Cage aux Folles by Jerry Herman \\
\hline 1983 & Sunday Bloody Sunday & $\mathrm{U} 2$ \\
\hline 1983 & Wade the Water to My Knees & $\begin{array}{l}\text { adaptation of "Wade in the Water" by McIntosh } \\
\text { County Shouters }\end{array}$ \\
\hline 1984 & Come the Revolution & $\begin{array}{l}\text { from E.G.: A Musical Portrait of Emma } \\
\text { Goldman; words: Karen Ruoff Kramer; words } \\
\text { and music: Leonard Lehrman }\end{array}$ \\
\hline 1984 (published) & Freedom Is Coming & $\begin{array}{l}\text { collected by Anders Nyberg in South African } \\
\text { Freedom Songs }\end{array}$ \\
\hline 1984 & MLK & U2 \\
\hline 1984 & Pride (In the Name of Love) & $\mathrm{U} 2$ \\
\hline 1985 & They Killed Him & Kris Kristofferson \\
\hline 1985 & Your Daughters and Your Sons & Tommy Sands \\
\hline 1988 & Across the Lines & Tracy Chapman \\
\hline 1988 & Black Steel in the Hour of Chaos & Public Enemy \\
\hline 1988 & Bread and Roses & $\begin{array}{l}\text { words: James Oppenheim, 1911; music: John } \\
\text { Denver, } 1988\end{array}$ \\
\hline 1988 & Daybreak in Alabama & $\begin{array}{l}\text { words: Langston Hughes, 1940; music: Elie } \\
\text { Siegmeister }\end{array}$ \\
\hline 1988 & Fight the Power & Public Enemy \\
\hline 1988 & Fuck tha Police & N.W.A. \\
\hline
\end{tabular}




\begin{tabular}{|c|c|c|}
\hline 1988 & I'm Gon' Stand & Sweet Honey in the Rock \\
\hline 1988 & Talkin' 'bout a Revolution & Tracy Chapman \\
\hline $1988-9$ & Nothin' [Nothing] New & Gloria Estefan \\
\hline 1989 & Sister Rosa & The Neville Brothers \\
\hline 1989 & They All Sang "Bread and Roses" & Si Kahn \\
\hline 1989 & We Didn't Start the Fire & Billy Joel \\
\hline 1990 & Anti-Nigger Machine & Public Enemy \\
\hline 1990 & Black and Proud & Tragedy Khadafi \\
\hline 1990 & Burn Baby Burn & 2 Black 2 Strong \\
\hline 1990 (recorded) & Ninety-Nine and a Half Won't Do & $\begin{array}{l}\text { Carlton Reese (Sing For Freedom: The Story of } \\
\text { the Civil Rights Movement Through Its Songs, } \\
\text { Smithsonian Folkways) }\end{array}$ \\
\hline 1991 & By the Time I Get to Arizona & Public Enemy \\
\hline 1991 & I Have Seen Freedom & Si Kahn \\
\hline 1991 & Just a Friendly Game of Baseball & Main Source \\
\hline 1991 & No Nose Job & Digital Underground \\
\hline 1991 & Old Jack Davey & Si Kahn \\
\hline 1991 & Optimistic & Sounds of Blackness \\
\hline 1992 & Cop Killer & Body Count \\
\hline 1992 & Wake Up & Rage Against the Machine \\
\hline 1992 & Who Got the Camera & Ice Cube \\
\hline 1992 & Killing in the Name & Rage Against the Machine \\
\hline 1993 & Just Another Day... & Queen Latifah \\
\hline 1993 & None of Us Are Free & Barry Mann, Cynthia Weil, and Brenda Russell \\
\hline 1993 & Putting Up Resistance & Beres Hammond \\
\hline 1993 & Sound of da Police & KRS-One \\
\hline 1993 & U.N.I.T.Y. & Queen Latifah \\
\hline 1994 & I Love Everybody & Lyle Lovett \\
\hline 1994 & Like a King & Ben Harper \\
\hline 1996 & Bulls on Parade & Rage Against the Machine \\
\hline 1996 & Make Them Hear You & from Ragtime; Stephen Flaherty and Lynn Ahrens \\
\hline 1996 & The Pride & Chuck D \\
\hline 1996 & They Don't Care About Us & Michael Jackson \\
\hline 1996 & To Live and Die in L.A. & Makaveli [Tupac Shakur] \\
\hline 1996 & White Man'z World & Makaveli [Tupac Shakur] \\
\hline 1998 & Changes & Tupac Shakur \\
\hline 1998 & Daybreak in Alabama & $\begin{array}{l}\text { words: Langston Hughes, 1940; music: Ricky Ian } \\
\text { Gordon }\end{array}$ \\
\hline 1998 & Forgive Them Father & Lauryn Hill \\
\hline 1998 & Lincoln's Army & The Irish Rovers \\
\hline 1999 & Colorgate & Julie Gibbons Kinscheck \\
\hline 1999 & Freedom Song & CosmoPop \\
\hline 1999 & Mathematics & Mos Def \\
\hline 1999 & Umi Says & Mos Def \\
\hline 2000 & Can U C the Pride in the Panther? & Mos Def (based on poetry by Tupac Shakur) \\
\hline 2000 & A Tree Never Grown & Mos Def and Talib Kweli \\
\hline 2000 & Welcome to S.N.C.C. & James Horner \\
\hline $\begin{array}{l}\text { pre-2001 (exact } \\
\text { date unknown) }\end{array}$ & We Shall Not Give Up the Fight & Anti-apartheid song \\
\hline
\end{tabular}




\begin{tabular}{|c|c|c|}
\hline 2001 & American Skin (41 Shots) & Bruce Springsteen \\
\hline 2001 & Burn Baby Burn & Ash \\
\hline 2002 & $\begin{array}{l}\text { A Dream Deferred [a.k.a. } \\
\text { Harlem] }\end{array}$ & $\begin{array}{l}\text { words: Langston Hughes, 1951; music: Leonard } \\
\text { Lehrman }\end{array}$ \\
\hline 2002 & My Block & Scarface \\
\hline 2003 & Everyone's a Little Bit Racist & from Avenue $Q$ by Robert Lopez and Jeff Marks \\
\hline 2003 & Song of Freedom & Marty Sampson \\
\hline 2004 & Ghetto Gospel & $\begin{array}{l}\text { Tupac Shakur (posthumous release of } 1992 \\
\text { recording) }\end{array}$ \\
\hline 2004 & Hold Fast to Dreams & $\begin{array}{l}\text { words: Langston Hughes, 1922; music: Dave } \\
\text { Brubeck }\end{array}$ \\
\hline 2004 & I Just Wanna Live & Good Charlotte \\
\hline 2004 & 'Merican & The Descendents \\
\hline 2004 & Rise Above It & Afro Celt Sound System \\
\hline 2005 & Freedom Song & Luc and the Lovingtons \\
\hline 2005 & Hold Fast to Dreams & $\begin{array}{l}\text { words: Langston Hughes, 1922; music: Leonard } \\
\text { Lehrman }\end{array}$ \\
\hline 2005 & Up to the Mountain & Patty Griffin \\
\hline 2006 & Ballad of Martin Luther King & Michael Borkson \\
\hline 2006 & A Dream & Common \\
\hline 2007 & Be the Change & Arlon Bennett \\
\hline 2007 & Black Boys & Bashy \\
\hline 2007 & Freedom Ain't Free & Brother Ali \\
\hline 2007 & $\begin{array}{l}\text { From the Plantation to the } \\
\text { Penitentiary }\end{array}$ & Wynton Marsalis \\
\hline 2007 & Harder Than You Think & Public Enemy \\
\hline 2007 & Same Thing & Flobots \\
\hline 2008 & By My Silence & Ellen Bukstel and Nick Annis \\
\hline 2008 & Hold Fast to Dreams & $\begin{array}{l}\text { words: Langston Hughes, 1922; music: André J. } \\
\text { Thomas }\end{array}$ \\
\hline 2008 & If You're Out There & John Legend \\
\hline 2008 & $\begin{array}{l}\text { Sharing a Gibson with Martin } \\
\text { Luther King, Jr. }\end{array}$ & Kurt Wagner \\
\hline 2008 & Take It from Dr. King & Pete Seeger \\
\hline 2008 & We Are the Ones & will.i.am \\
\hline 2008 & Yes We Can & $\begin{array}{l}\text { words: "Barack Obama concession speech at New } \\
\text { Hampshire primary"; music: will.i.am }\end{array}$ \\
\hline 2009 & John Brown & David Rovics \\
\hline 2010 & $\begin{array}{l}\text { Long Island Is Just Not Long } \\
\text { Enough }\end{array}$ & $\begin{array}{l}\text { words: Marcia McNair, 2009; music: Leonard } \\
\text { Lehrman }\end{array}$ \\
\hline 2010 & 1960 What? & Gregory Porter \\
\hline 2010 & Not Afraid & Eminem \\
\hline 2010 & Power & Kanye West \\
\hline $\begin{array}{l}2011 \text { (exact date } \\
\text { unknown) }\end{array}$ & About Face & Maggie Martin \\
\hline 2011 & For Gene Debs & Anne Feeney \\
\hline 2011 & Let America Be America Again & $\begin{array}{l}\text { words: Langston Hughes, 1935; music: Leonard } \\
\text { Lehrman }\end{array}$ \\
\hline 2011 & My Name Is Emmett Till & Emmylou Harris \\
\hline 2011 & No Church in the Wind & Jay-Z and Kanye West \\
\hline
\end{tabular}




\begin{tabular}{|c|c|c|}
\hline 2011 & Nothin' New & Ab-Soul \\
\hline 2011 & The People Will Rise & Nelini Stamp \\
\hline 2011 & So Beautiful or So What & Paul Simon \\
\hline 2011 & $\begin{array}{l}\text { We Are the Ones (We've Been } \\
\text { Waiting For) }\end{array}$ & Melissa Etheridge \\
\hline $\begin{array}{l}2005-12 \text { (exact } \\
\text { date unknown) }\end{array}$ & Spirit Found Us & $\begin{array}{l}\text { words: David Schiffman, 2005; music: Lisa G. } \\
\text { Littlebird }\end{array}$ \\
\hline 2012 & $\begin{array}{l}\text { Around My Way (Freedom Ain't } \\
\text { Free) }\end{array}$ & Lupe Fiasco \\
\hline 2012 & Black Rage & $\begin{array}{l}\text { Lauryn Hill (tune: "My Favorite Things" from } \\
\text { The Sound of Music, 1960; music: Richard } \\
\text { Rodgers) }\end{array}$ \\
\hline 2012 & Blood from Stones & Si Kahn \\
\hline 2012 & Break the Chain & Tena Clark and Tim Heintz \\
\hline 2012 & Land of the Free & Esperanza Spalding \\
\hline 2012 & Vann Plantation & Si Kahn \\
\hline 2012 & We Are Alive & Bruce Springsteen \\
\hline 2013 & $\begin{array}{l}\text { Freedom Song (They'll Never } \\
\text { Take Us Down) }\end{array}$ & Neil Diamond \\
\hline 2013 & Hell You Talmbout & Janelle Monáe \\
\hline 2013 & Hold Fast to Dreams & $\begin{array}{l}\text { words: Langston Hughes, 1922; music: Susan } \\
\text { LaBarr }\end{array}$ \\
\hline 2013 & New Slaves & Kanye West \\
\hline 2013 & One & India.Arie \\
\hline 2014 & Be Free & J. Cole (about Michael Brown) \\
\hline 2014 & The Charade & D'Angelo \\
\hline 2014 & Don't Shoot & The Game \\
\hline 2014 & $\begin{array}{l}\text { Everybody Ought To Know } \\
\text { What Freedom Is }\end{array}$ & $\begin{array}{l}\text { Ysaye Barnwell (adapted from hymn by Harry } \\
\text { Dixon Loes, 1940) }\end{array}$ \\
\hline 2014 & Glory & from Selma; Common and John Legend \\
\hline 2014 & Hands Up & Vince Staples \\
\hline 2014 & Marching on Ferguson & Tom Morello \\
\hline 2014 & One Love & Elle Varner \\
\hline 2014 & Rollcall for Those Absent & Ambrose Akinmusire \\
\hline 2014 & Unity & TheFatRat (Christian Büttner) \\
\hline 2014 & We Are Strong & Lil Bibby \\
\hline 2014 & We Gotta Pray & Alicia Keys \\
\hline 2014 & White Privilege & Macklemore and Ryan Lewis \\
\hline 2015 & All Black & Raftaar \\
\hline 2015 & Alright & Kendrick Lamar \\
\hline 2015 & Baltimore & Prince \\
\hline 2015 & The Blacker the Berry & Kendrick Lamar \\
\hline 2015 & Chains & Usher \\
\hline 2015 & Cry No More & Rhiannon Giddens \\
\hline 2015 & Freedom Is a Funny Thing & Malik \& The O.G's \\
\hline 2015 & $\begin{array}{l}\text { Freedom Side-Which Side Are } \\
\text { You On? }\end{array}$ & $\begin{array}{l}\text { Movement for Black Lives (adaptor unknown) } \\
\text { (tune: Which Side Are You On?) }\end{array}$ \\
\hline 2015 & Hand in Hand & Jayanthi Kyle and Wes Burdine \\
\hline 2015 & I Am Not Afraid & Peace Poets \\
\hline
\end{tabular}




\begin{tabular}{|c|c|c|}
\hline 2015 & $\begin{array}{l}\text { It's Not So Very Far from the } \\
\text { Mississippi Clay }\end{array}$ & Greg Artzner and Terry Leonino \\
\hline 2015 & King Kunta & Kendrick Lamar \\
\hline 2015 & $\begin{array}{l}\text { We Will Remember: Song for } \\
\text { Michael Brown }\end{array}$ & $\begin{array}{l}\text { Raging Grannies, Vicki Ryder, NJ Solidarity } \\
\text { Singers, Roseanne DePasquale (tune: "The Water } \\
\text { Is Wide") }\end{array}$ \\
\hline 2015 & Wesley's Theory & Kendrick Lamar \\
\hline 2016 & Better Days & Victoria Monét and Ariana Grande \\
\hline 2016 & Black America Again & Common \\
\hline 2016 & F.U.B.U. & Solange \\
\hline 2016 & Formation & Beyoncé \\
\hline 2016 & Freedom & Beyoncé \\
\hline 2016 & Freedom Freestyle & Joe Budden \\
\hline 2016 & Hands Up & Daye Jack and Killer Mike \\
\hline 2016 & Here in My Hands & Amy Wadge \\
\hline 2016 & How Many & Miguel \\
\hline 2016 & $\begin{array}{l}\text { I'm Gonna Lift My } \\
\text { Sister/Brother Up }\end{array}$ & Faya Rose Toure \\
\hline 2016 & I'm Gonna Walk It With You & Brian Claflin and Ellie Grace \\
\hline 2016 & Isombard & $\begin{array}{l}\text { words: "next to of course god america i" by E. E. } \\
\text { Cummings, 1926; music: Declan McKenna }\end{array}$ \\
\hline 2016 & Last Ones & Amaal Nuux \\
\hline 2016 & Lead with Love & Melanie DeMore \\
\hline 2016 & A Lot of Love & Chris Brown \\
\hline 2016 & $\begin{array}{l}\text { Mi Unica (No human being will } \\
\text { ever be illegal) }\end{array}$ & Peace Poets \\
\hline 2016 & No Justice No Peace & Z-ro \\
\hline 2016 & Nobody Speak & DJ Shadow \\
\hline 2016 & Sad News & Swizz Beatz \\
\hline 2016 & (Same Old) Rich Man's Strategy & Peace Poets \\
\hline 2016 & Sandra's Smile & Blood Orange \\
\hline 2016 & 16 Shots & Stefflon Don \\
\hline 2016 & Spiritual & Jay Z \\
\hline 2016 & Thieves! Screamed the Ghost & Run The Jewels \\
\hline 2017 & Bleed the Same & Mandisa \\
\hline 2017 & Freedom Highway & Rhiannon Giddens \\
\hline 2017 & Harriet-The Conductor & Lak \\
\hline 2017 & i can't breathe & Bea Miller \\
\hline 2017 & 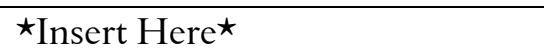 & VANT \\
\hline 2017 & Land of the Free & Joey Bada $\$ \$$ \\
\hline 2017 & Nothin' New & 21 Savage \\
\hline 2017 & $\begin{array}{l}\text { People Gonna Rise Like the } \\
\text { Water }\end{array}$ & Peace Poets \\
\hline 2017 & Praying with Our Feet & Rabbi Joe Black and Steven Brodsky \\
\hline 2017 & Room for the Refugee & Tom Bias \\
\hline 2017 & Sing Out/March On & Joshuah Campbell \\
\hline 2017 & The Story of O.J. & Jay-Z \\
\hline 2017 & Tear Down That Wall & Bright Light Social Hour \\
\hline 2017 & Ten Thousand Bridges & Greg Artzner and Terry Leonino \\
\hline
\end{tabular}




\begin{tabular}{|c|c|c|}
\hline 2017 & (There Are) More Waters Rising & Saro Lynch Thomason \\
\hline 2017 & We Will Rise Together & Hali Hammer \\
\hline 2018 & Blam & Georgia Anne Muldrow \\
\hline 2018 & Blue Lights & Jorja Smith \\
\hline 2018 & Calling All [the] Warriors & Dina Erie \\
\hline 2018 & Cops Shot the Kid & Nas \\
\hline 2018 & Don't Don't Do It! & Jay Rock \\
\hline 2018 & $\begin{array}{l}\text { A Dream Deferred [a.k.a. } \\
\text { Harlem] }\end{array}$ & $\begin{array}{l}\text { words: Langston Hughes, 1951; music: Ricky Ian } \\
\text { Gordon }\end{array}$ \\
\hline 2018 & Freedom Ain't Free & Anika Whitfield \\
\hline 2018 & Hold Fast to Dreams & $\begin{array}{l}\text { words: Langston Hughes, 1922; music: Joel } \\
\text { Thompson }\end{array}$ \\
\hline 2018 & $\begin{array}{l}\text { I Have Seen Freedom Being } \\
\text { Born }\end{array}$ & Joe Jencks \\
\hline 2018 & $\begin{array}{l}\text { I Wanna Be Ready for Change to } \\
\text { Come }\end{array}$ & Charon Hribar (tune: "I Wanna Die Easy") \\
\hline 2018 & Kapernick Effect & JAG \\
\hline 2018 & King's Dead & Jay Rock \\
\hline 2018 & Love It If We Made It & The 1975 \\
\hline 2018 & Rise Up & Charon Hribar and Jose Vasquez \\
\hline 2018 & Shake Injustice Off & Yara Allen (tune: "Shake the Devil Off") \\
\hline 2018 & 6 Summers & Anderson .Paak \\
\hline 2018 & $\begin{array}{l}\text { Somebody's Hurting My } \\
\text { Brother [My Sister] [Our } \\
\text { People] }\end{array}$ & Yara Allen \\
\hline 2018 & $\begin{array}{l}\text { The Stone Throwers (Gone in a } \\
\text { Blink) }\end{array}$ & Shad \\
\hline 2018 & $\begin{array}{l}\text { There's a Moral Revolution } \\
\text { Going On }\end{array}$ & Ruth MacKenzie \\
\hline 2018 & This Is America & Childish Gambino [Donald Glover] \\
\hline 2018 & $\begin{array}{l}\text { Un Zol vi Veyt (Dos Naye Lid } \\
\text { [The New Song]) }\end{array}$ & words: Avrom Reisen; music: Louis Schwartz \\
\hline 2018 & Unsettling Force & Luke Nephew \\
\hline 2018 & We are the Protectors & Peace Poets \\
\hline $\begin{array}{l}2018 \text { (exact date } \\
\text { unknown) }\end{array}$ & We Have Come & Luke Neighbor \\
\hline 2018 & Why Are the Guns Still Firing? & Joe Jencks \\
\hline 2019 & Be a Pain & Alastair Moock \\
\hline 2019 & Bells (Ring Loudly) & Terri Lyne Carrington \\
\hline 2019 & Don't You Wanna Vote & Yara Allen (tune: "Don't You Wanna Go") \\
\hline 2019 & $\begin{array}{l}\text { In My Heart (I Want to Fight } \\
\text { for...) }\end{array}$ & Composer unknown \\
\hline 2019 & Land of the Free & The Killers \\
\hline 2019 & Mamma's Cryin' Long & Rhiannon Giddens \\
\hline 2019 & Nina & Rapsody \\
\hline 2019 & Organize, Organize, Organize & Composer unknown (adapted by Charon Hribar) \\
\hline 2019 & Our Hands & Lu Aya and Charon Hribar \\
\hline 2019 & Revealing the Truth & Lu Aya \\
\hline 2019 & Strange Things & $\begin{array}{l}\text { Composer unknown (adapted by Karen Porter } \\
\text { and VT Solidarity Singers) }\end{array}$ \\
\hline
\end{tabular}




\begin{tabular}{|c|c|c|}
\hline 2019 & $\begin{array}{l}\text { There's a New World Coming } \\
\text { Over }\end{array}$ & Bernice Johnson Reagon \\
\hline 2020 & All That Matters & Dana Vance \\
\hline 2020 & The Bigger Picture & Lil Baby \\
\hline 2020 & Black Like Me & Mickey Guyton \\
\hline 2020 & body cast & Dua Saleh \\
\hline 2020 & $\begin{array}{l}\text { Bread, Roses and RBG's Last } \\
\text { Wish }\end{array}$ & $\begin{array}{l}\text { words: Leonard Lehrman (on Ruth Bader } \\
\text { Ginsburg) (music: "Bread and Roses" by Mimi } \\
\text { Fariña, 1974) }\end{array}$ \\
\hline 2020 & BREATHE & Adrienne Danrich and Drew Hemenger \\
\hline 2020 & Cops with Guns are the Worst!!! & Snotty Nose Rez Kids \\
\hline 2020 & Days of Decision & $\begin{array}{l}\text { (adapted for John Lewis by Roseanne } \\
\text { DePasquale) (based on "Days of Decision," Phil } \\
\text { Ochs, 1965) }\end{array}$ \\
\hline 2020 & Freedom Is A Constant Song & Si Kahn \\
\hline 2020 & Frightened & Dave Williams \\
\hline 2020 & $\begin{array}{l}\text { Hallelujah; I'm On My Journey; } \\
\text { Ride the Chariot }\end{array}$ & excerpts from opera Freedom Ride by Dan Shore \\
\hline 2020 & I Can't Breathe & H.E.R. \\
\hline 2020 & Imaginary Graduation Speech & Leonard Lehrman \\
\hline 2020 & JU\$T & $\begin{array}{l}\text { Run The Jewels, Zack De La Rocha, and Pharrell } \\
\text { Williams }\end{array}$ \\
\hline 2020 & Our Turn Now & Len Seligman (on John Lewis) \\
\hline 2020 & Pig Feet & Terrace Martin \\
\hline 2020 & Pity the Nation & $\begin{array}{l}\text { words: Lawrence Ferlinghetti, 2007; music: } \\
\text { Leonard Lehrman }\end{array}$ \\
\hline 2020 & Say Can You See & Scott Cook \\
\hline 2020 & Scottie Beam & Freddie Gibbs and The Alchemist \\
\hline 2020 & Sin Aire [Without Air] & Rafa Pabön \\
\hline 2020 & $\begin{array}{l}\text { Stay in Place, Stay Alive, } \\
\text { Organize }\end{array}$ & $\begin{array}{l}\text { Natalie Robinson and Jessica Petersen (tune: } \\
\text { "Organize, Organize, Organize") }\end{array}$ \\
\hline 2020 & Sweeter & Leon Bridges \\
\hline 2020 & Thinking Outside the Box & George Wallace and Leonard Lehrman \\
\hline 2020 & Underdog & $\begin{array}{l}\text { Alicia Keys, Johnny McDaid, Ed Sheeran, Amy } \\
\text { Wadge, Jonny Coffer, and Foy Vance }\end{array}$ \\
\hline 2020 & Walking in the Snow & Run The Jewels \\
\hline 2020 & What Matters & Leonard Lehrman \\
\hline
\end{tabular}




\section{Acknowledgements}

Acknowledgments are in order to the authors of various online lists posted by The Progressive, The Nation, Rolling Stone, the Poor People's March, and Students4Khazei on Spotify; along with Zalmen Mlotek, Si Kahn, Philip Aaberg, Bennet Zurofsky \& the Solidarity Singers of the New Jersey Industrial Union Council, and Forward March's Dave Williams. Also to Ralph Locke and Michael Beckerman, who encouraged me to submit this to Music \& Politics. 